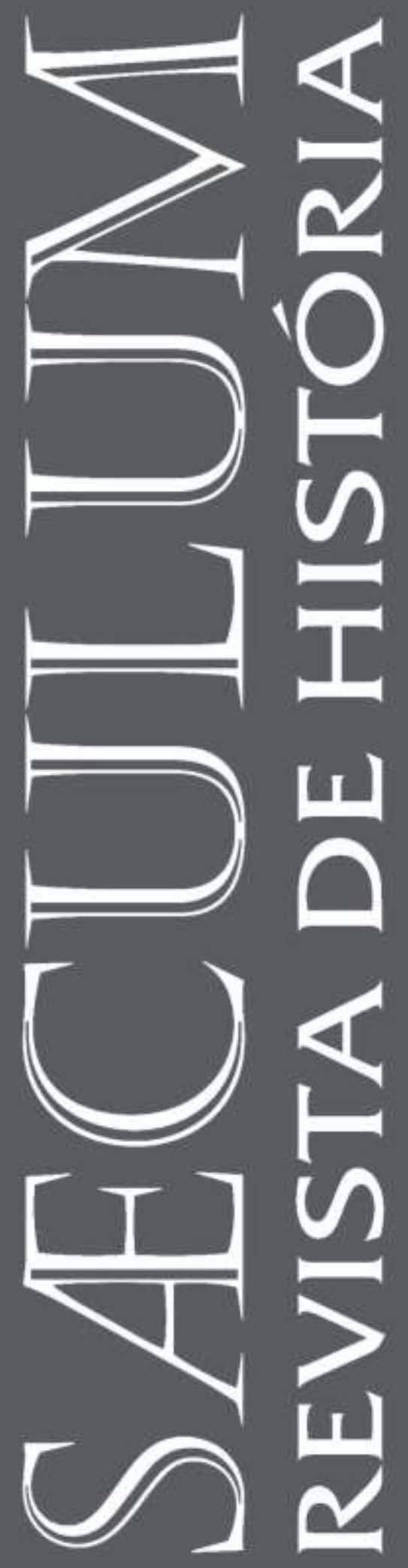

AV $A=m$ in

raphos. if in 4 , sw Ant C N (3)

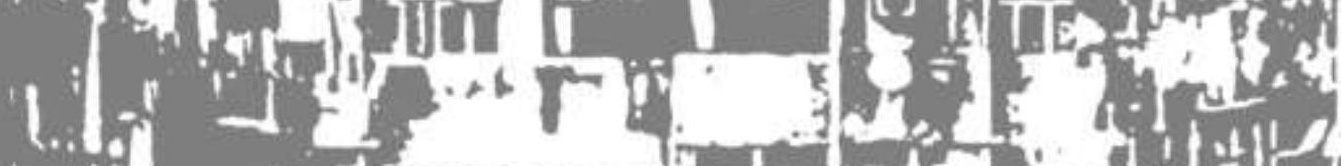
in 1 igr

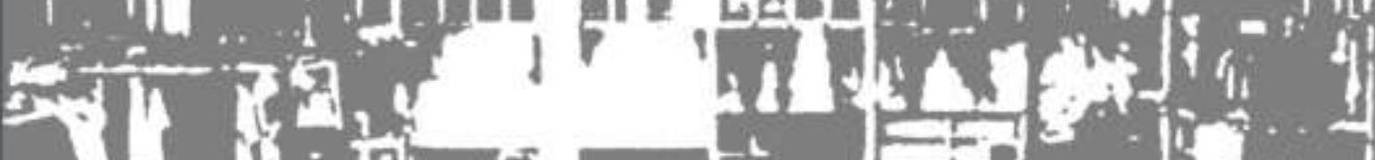
$4 \mathrm{~N}^{2} \mathrm{~s}$ - 14 ind 1 en

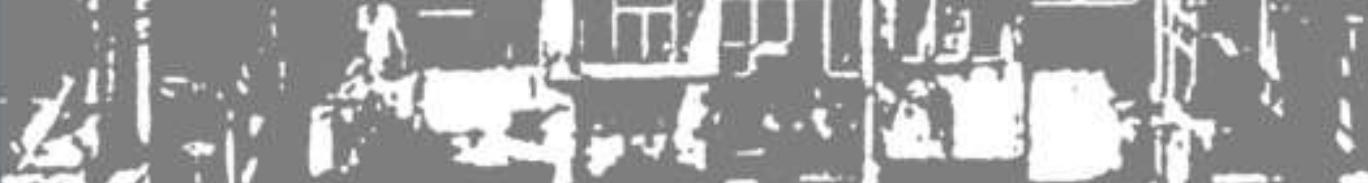
(N)

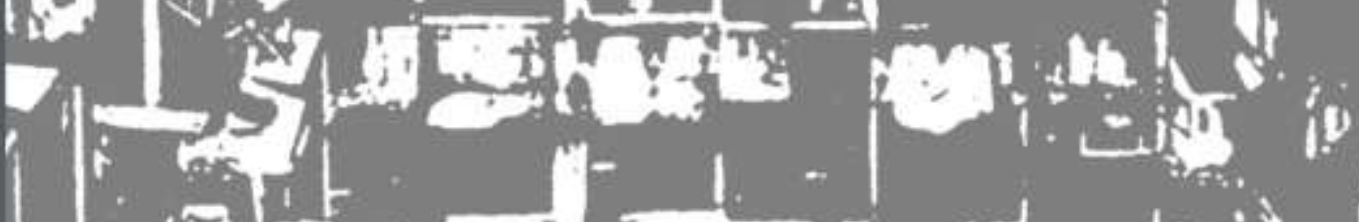
(1)

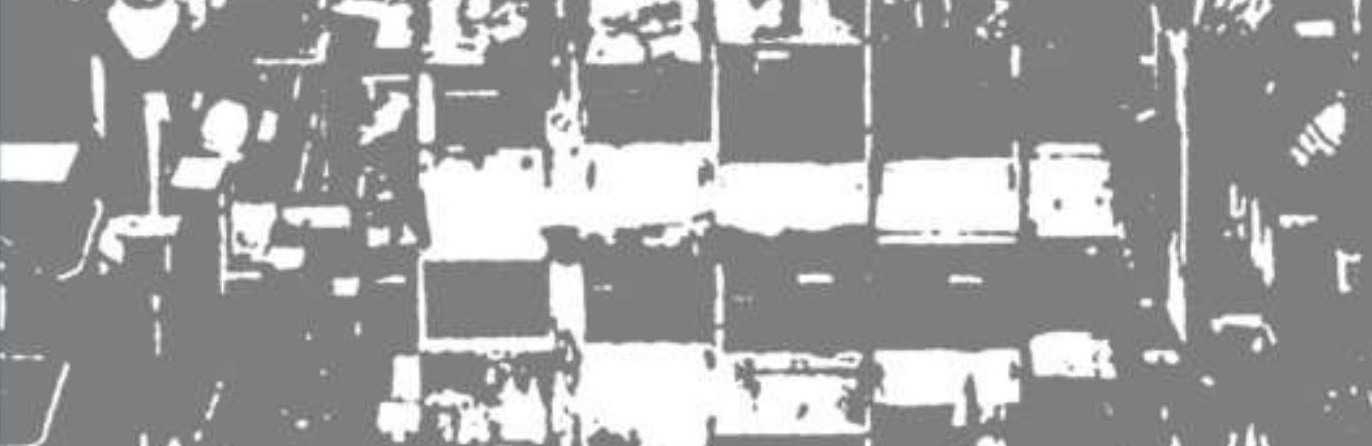
JUL./DEZ. 2020
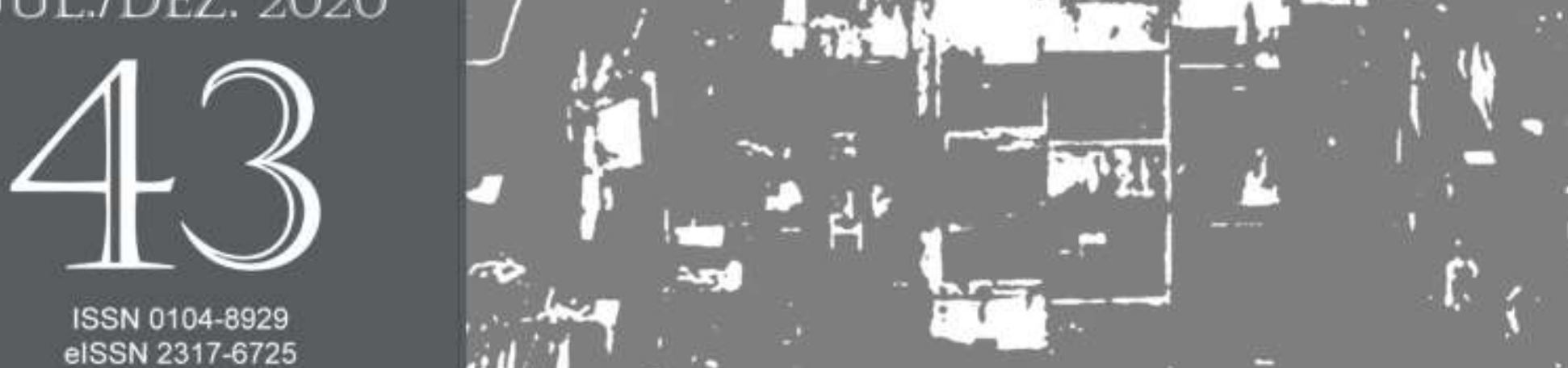


\title{
SÆCULUM
}

\section{REVISTA DE HISTÓRIA}

\author{
Vol. 25 - Número 43 - jul./dez. 2020
}

ISSNe 2317-6725

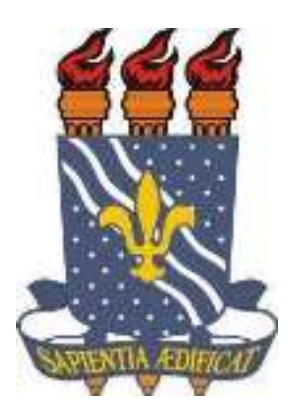

\section{UNIVERSIDADE FEDERAL DA PARAÍBA}

Reitora: Margareth de Fátima Formiga Melo Diniz Vice-Reitora: Bernardina Maria Juvenal Freire de Oliveira

\section{PRÓ-REITORIA DE PESQUISA}

Pró-Reitor: Isac Almeida de Medeiros

PRÓ-REITORIA DE PÓS-GRADUAÇÃo

Pró-Reitora: Maria Luiza Pereira Alencar M. Feitosa

\section{CENTRO DE CIÊNCIAS HUMANAS, LETRAS E ARTES}

Diretora: Mônica Nóbrega

Vice-Diretor: Rodrigo Freire de Carvalho e Silva

\section{DEPARTAMENTO DE HISTÓRIA}

Chefe: Fernando Cauduro Pureza

Subchefe: Monique Guimarães Cittadino 


\section{PROGRAMA DE PÓS-GRADUAÇÃO EM HISTÓRIA}

Coordenador: Tiago Bernardon de Oliveira

Vice-coordenador: Paulo Giovani Antonino Nunes

\section{COMISSÃO EDITORIAL - S/ECULUM}

Ana Maria Veiga (presidente)

Ana Beatriz Ribeiro Barros Silva

Ângelo Emílio da Silva Pessoa

Ariane Norma de Menezes Sá

Carla Mary S. Oliveira

Cláudia Engler Cury

Damião de Lima

Fernando Cauduro Pureza

Guilherme Queiroz de Souza (editor)

Lúcio Flávio Sá Peixoto de Vasconcelos

Mozart Vergetti de Menezes

Paulo Roberto de Azevedo Maia

Raimundo Barroso Cordeiro

Serioja Rodrigues Cordeiro Mariano

Telma Dias Fernandes

Tiago Bernardon de Oliveira 


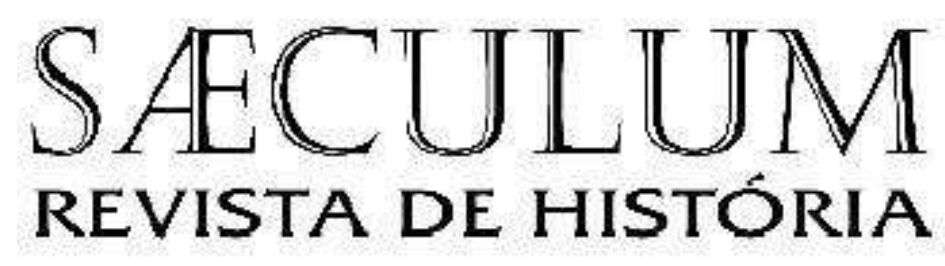

\title{
EDITORES RESPONSÁVEIS
}

\author{
Ana Maria Veiga - UFPB \\ Guilherme Queiroz de Souza - UFPB
}

EDITOR ASSISTENTE

Jair Zandoná - UFSC

SECRETARIA

Letícia Helen Silva Teles

Zênia Chaves Araújo de Melo

(mestrandas do PPGH/UFPB)

\section{Departamento de História \\ Programa de Pós-Graduação em História Universidade Federal da Paraíba}

\author{
CENTRO DE CIÊNCIAS HUMANAS, LETRAS E ARTES \\ Campus Universitário - Conjunto Humanístico - Bloco V \\ Castelo Branco - João Pessoa - Paraỉba - CEP 58051-970 - Brasil \\ Fone/ Fax: +55 (83) 3216-7915 - e-mail: saeculum@cchla.ufpb.br \\ Sítio eletrônico: https://periodicos.ufpb.br/index.php/srh
}

\section{Copyright (C) 1995-2020 - DH/PPGH/UFPB \\ ISSN 0104-8929 - ISSNs 2317-6725}

Capa, Projeto Gráfico e Editoração Eletrônica:

Ana Maria Veiga, Carla Mary S. Oliveira, Sônia Weidner Maluf

Arte do vol. 25, n. 43: montagem sobre o tema biopolíticas e necropolíticas Autora: Sônia Weidner Maluf

Este periódico está licenciado sob a Licença Creative Commons CC-BY.

Com essa licença você pode compartilhar, adaptar, criar para qualquer fim, desde que atribua a autoria da obra. 
Periódico avaliado no estrato QUALIS B1 (A3 no Qualis Referência 2017-2018) na área de História pela Capes

\section{CONSELHO EDITORIAL}

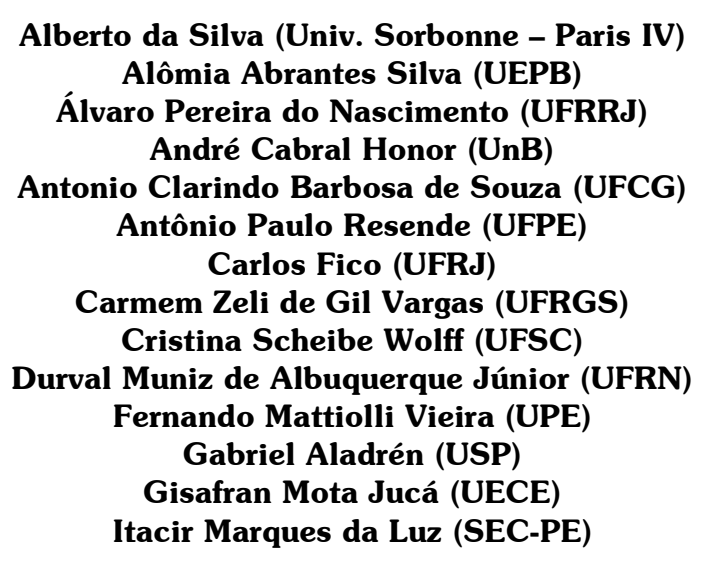

\author{
Itamar Freitas (UFS) \\ Jorge Ferreira (UFF) \\ José Miguel Arias Neto (UEL) \\ Lina Maria Brandão de Aras (UFBA) \\ Luiz Geraldo Silva (UFPR) \\ Maria de Deus Beites Manso (Univ. Évora) \\ Pedro Paulo Funari (UNICAMP) \\ Peter Mainka (Univ. de Wüzburg) \\ Ricardo Pinto de Medeiros (UFPE) \\ Sílvia Regina Ferraz Petersen (UFRGS) \\ Tania Bessone (UERJ) \\ Thereza Baumann (UFRJ) \\ Valdemir Zamparoni (UFBA)
}

\section{MISSÃO DA REVISTA}

Sæculum - Revista de História é publicada pelo Departamento de História da UFPB desde 1995 e, a partir de 2004, passou a ser também o periódico do Programa de Pós-Graduação em História da mesma universidade. Sua frequência é semestral, e se trata de uma revista voltada à divulgação e debate de pesquisas no campo da História e da Cultura Histórica e suas diversas interfaces, abrindo espaço para pesquisadores do Brasil e do exterior.

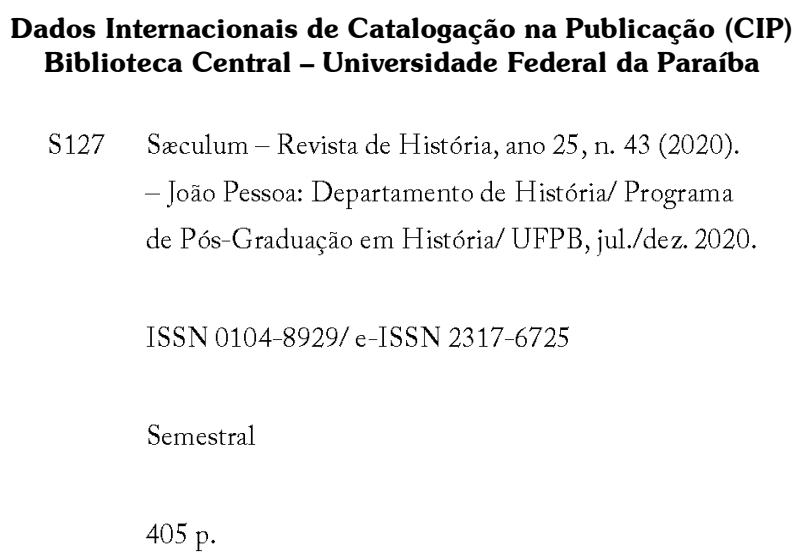


A Comissão Editorial da Sæculum agradece aos seguintes colegas, que atuaram como pareceristas ad hoc na presente edição:

Adriano Toledo Paiva, Universidade Federal de Minas Gerais

Afonso de Alencastro Graça Filho, Universidade Federal de São João Del-Rei

Alcilene Cavalcante, Universidade Federal de Goiás

Alexandre Andrade da Costa, Fundação Educacional de Fernandópolis

Antônio de Pádua Bosi, Universidade Estadual do Oeste do Paraná

Braz Batista Vas, Universidade Federal do Tocantins

Camila Rodrigues Jourdan, Universidade do Estado do Rio de Janeiro

Carlos de Oliveira Malaquias, Universidade Federal de Sergipe

Caroline Moreira Vieira Dantas, Universidade do Estado do Rio de Janeiro

Daniel Precioso, Universidade Estadual de Goiás

Débora Breder, Universidade Católica de Petrópolis

Diego Souza de Paiva, Núcleo de Cultura da Universidade Federal do Rio Grande do Norte

Eduardo Henrique de Lima Guimarães, Universidade Federal da Paraíba

Eduardo Romero de Oliveira, Universidade Estadual Paulista Júlio de Mesquita Filho

Erivaldo Fagundes Neves, Universidade Estadual de Feira de Santana

Fabiana Comerlato, Universidade Federal do Recôncavo da Bahia

Fabiana da Silva Viana, Instituto Cecília Meireles

Fátima Saionara Leandro Brito, Instituto Federal de Educação, Ciência e Tecnologia do Sul de Minas Gerais

Fernanda Cristina da Encarnação dos Santos, Universidade Federal do Amapá

Flávia Cristina Silveira Lemos, Universidade Federal do Pará

Gilmar Arruda, Universidade Estadual de Londrina

Guilherme Amaral Luz, Universidade Federal de Uberlândia

Isabela Candeloro Campoi, Universidade Estadual do Paraná

Ivanice Teixeira Silva Ortiz, Universidade do Estado da Bahia

Joana D'Arc de Sousa Lima, Universidade Federal de Pernambuco

Joelma Santos da Silva, Instituto Federal de Educação, Ciência e Tecnologia do Maranhão

Johny Santana de Araújo, Universidade Federal do Piauí

Kátia Sausen da Motta, Universidade Federal do Espírito Santo

Kelly Eleutério Machado Oliveira, Universidade Federal de Ouro Preto

Laurindo Mekie Pereira, Universidade Estadual de Montes Claros

Leicy Francisca da Silva, Universidade Estadual de Goiás

Lia Calabre, Fundação Casa de Rui Barbosa

Luiz Antonio da Silva Teixeira, Universidade Estácio de Sá

Luiz Otávio Ferreira, Fundação Oswaldo Cruz

Marcília Gama da Silva, Universidade Federal Rural de Pernambuco

Maria da Vitória Barbosa Lima, Universidade Estadual do Piauí

Maria de Fátima Oliveira, Universidade Estadual de Goias

Martinho Guedes dos Santos Neto, Universidade Federal da Paraíba

Maurício Gonçalves Margalho, Secretaria de Estado de Educação - RJ

Paloma Ferreira Coelho Silva, Pontifícia Universidade Católica de Minas Gerais

Regina Mendes de Araújo, Instituto Federal do Norte de Minas

Reginaldo Cerqueira Sousa, Universidade Federal do Sul e Sudeste do Pará

Ricardo dos Santos Batista, Universidade do Estado da Bahia/Universidade de São Paulo

Ricardo Figueiredo de Castro, Universidade Federal do Rio de Janeiro

Rita de Cássia Dias Pereira de Jesus, Universidade Federal do Recôncavo Baiano

Silvia Maria Fávero Arend, Universidade do Estado de Santa Catarina

Sílvia Maria Jardim Brügger, Universidade Federal de São João Del-Rei

Sônia Weidner Maluf, Universidade Federal de Santa Catarina

Surya Aaronovich Pombo de Barros, Universidade Federal da Paraíba

Tânia Maria Tavares Bessone da Cruz Ferreira, Universidade do Estado do Rio de Janeiro

Valéria Gomes Costa, Instituto Federal de Pernambuco

Virgílio Coelho de Oliveira Júnior, Centro Federal de Educação Tecnológica de Minas Gerais

Wilma de Nazaré Baía Coelho, Universidade Federal do Pará 


\section{Sumário}

\section{DOSSIÊ: A NOVA HISTÓRIA (BIO)POLÍTICA: SOBRE AS CAPTURAS E AS RESISTÊNCIAS}

A nova história (bio)política: sobre as capturas e as resistências

Telma Dias Fernandes

Elisa Mariana de Medeiros Nóbrega

Biopolítica na epidemia de Covid-19 no Brasil (2020): uma análise das ações de combate à doença e a oposição antiquarentena

Leandro Vilar Oliveira

O corpo da criança nas tramas da biopolítica: quando a filantropia se fez máquina de captura (Brasil 1940-1950) José dos Santos Costa Júnior

Experiência, infância, linguagem e acontecimento: a biopolítica de Giorgio Agamben e a Educação Pedro Angelo Pagni

Raphael Guazzelli Valerio

As instituições de reclusão e "controle" dos loucos na Parahyba do Norte entre 1889 e 1928

Edna Maria Nóbrega Araújo

Joedna Reis de Meneses

A Anormalidade nos Domínios Biopolíticos: “A Organização de Assistência aos Psicopatas de Pernambuco” (1931) Rafael Santana Bezerra

Segurança Nacional e Biopolítica: as práticas e os dispositivos de informação e vigilância da DOPS-PE Raphael Henrique Roma Correia

Rever a colonização, reler a biopolítica, almejar o poder: os bandeirantes paulistas nas minas do Serro do Frio e Vila do Príncipe, Minas Gerais, 1702-1720

Danilo Arnaldo Briskievicz

O fantasma do corpo: a sexualidade como dispositivo do biopoder em A pele que Habito (2011)

Paul Jardim Martins Afonso

\section{ARTIGOS}

Black American Colonization in the Brazilian Amazon: Colored bodies in motion Marcia Esteves Agostinho

A modéstia e a ostentação no vestuário das mulheres alforriadas na Vila de Pitangui (1750-1820) Ana Caroline Carvalho Miranda

Circulação de papéis: notícias e ideias pela América portuguesa (1808-1822) Augusto Henrique Assis Resende

“Que fiquemos livres da jurisdição dos bispos do Maranhão": os discursos por autonomia eclesiástica da Província do Piauí (1829-1838)

João Vitor Araújo Sales

Marcelo de Sousa Neto

"Em nome da pátria e da glória": a formação do 1.o Corpo de Voluntários da Pátria (Pernambuco, 1865) Wellington Barbosa da Silva

Leocádia e suas mortes: o confronto de narrativas de um crime hediondo no Alto Sertão da Bahia do final do século 
XIX

Fernando Donato Vasconcelos

“Não há mais grave, mais perigosa, e mais temível”: a sífilis na província da Parahyba (1860-1880) Serioja R. C. Mariano

O abastecimento de água no Brasil: uma prospecção bibliográfica no âmbito da historiografia Fabiano Quadros Rückert

“A coisa está ficando feia”: o consumo da carne de baleia no Brasil entre a História e Antropologia (1960-1963) Fernando Cauduro Pureza

Democracia(s)? Algumas considerações sobre abertura política de 1945 na (entre)vista de José Américo e no Manifesto Mineiro

Cintya Chaves

Corpos ultrajantes, discursos interditos: embates entre a indústria cinematográfica erótica e a censura federal durante a ditadura civil-militar brasileira

Romulo Gabriel de Barros Gomes

Os cárceres da Guanabara através do jornal Ultima Hora (1960-1961)

Mariana Dias Antonio

Os novos contornos do antissemitismo, a construção de imaginários pela mídia no Brasil e a escrita da História: singularidades e perigos

Ana Heloisa Molina

Helena Ragusa

Réquiem para uma historiadora negra: morte e invisibilidade de Beatriz Nascimento Elio Chaves Flores

\section{RESENHAS}

"Escrevo da periferia, não do centro": mulheres negras e experiências de racismo cotidiano Claudia Regina Nichnig 